\title{
TU/e EmonOWEN

\section{Uniform illumination rendering using an array of LEDs: a signal processing perspective}

\section{Citation for published version (APA):}

Yang, H., Bergmans, J. W. M., Schenk, T. C. W., Linnartz, J. P. M. G., \& Rietman, R. (2009). Uniform illumination rendering using an array of LEDs: a signal processing perspective. IEEE Transactions on Signal Processing, 57(3), 1044-1057. https://doi.org/10.1109/TSP.2008.2009269

DOI:

10.1109/TSP.2008.2009269

Document status and date:

Published: 01/01/2009

\section{Document Version:}

Publisher's PDF, also known as Version of Record (includes final page, issue and volume numbers)

\section{Please check the document version of this publication:}

- A submitted manuscript is the version of the article upon submission and before peer-review. There can be important differences between the submitted version and the official published version of record. People interested in the research are advised to contact the author for the final version of the publication, or visit the $\mathrm{DOI}$ to the publisher's website.

- The final author version and the galley proof are versions of the publication after peer review.

- The final published version features the final layout of the paper including the volume, issue and page numbers.

Link to publication

\section{General rights}

Copyright and moral rights for the publications made accessible in the public portal are retained by the authors and/or other copyright owners and it is a condition of accessing publications that users recognise and abide by the legal requirements associated with these rights.

- Users may download and print one copy of any publication from the public portal for the purpose of private study or research.

- You may not further distribute the material or use it for any profit-making activity or commercial gain

- You may freely distribute the URL identifying the publication in the public portal.

If the publication is distributed under the terms of Article 25fa of the Dutch Copyright Act, indicated by the "Taverne" license above, please follow below link for the End User Agreement:

www.tue.nl/taverne

Take down policy

If you believe that this document breaches copyright please contact us at:

openaccess@tue.nl

providing details and we will investigate your claim. 


\title{
Uniform Illumination Rendering Using an Array of LEDs: A Signal Processing Perspective
}

\author{
Hongming Yang, Student Member, IEEE, Jan W. M. Bergmans, Senior Member, IEEE, \\ Tim C. W. Schenk, Member, IEEE, Jean-Paul M. G. Linnartz, Senior Member, IEEE, and Ronald Rietman
}

\begin{abstract}
An array of a large number of LEDs will be widely used in future indoor illumination systems. In this paper, we investigate the problem of rendering uniform illumination by a regular LED array on the ceiling of a room. We first present two general results on the scaling property of the basic illumination pattern, i.e., the light pattern of a single LED, and the setting of LED illumination levels, respectively. Thereafter, we propose to use the relative mean squared error as the cost function to measure the uniformity of the realized illumination pattern, and provide an analysis for this cost function. Based on the analysis, the design of a basic illumination pattern is discussed. The performances of a few basic illumination patterns are compared, and an approach for optimizing the basic illumination pattern through a weighted combination of these basic patterns is also proposed. A weighted combination of Gaussian and raised-cosine functions is found to yield the best results. Finally, three basic regular grid shapes for an LED array are compared. The results show that $13 \%$ and $39 \%$ of LEDs can be saved for the same degree of uniformity, using the hexagonal instead of the rectangular and triangular grid, respectively.
\end{abstract}

Index Terms-Basic illumination pattern, LED illumination, regular grid, two-dimensional signal processing.

\section{INTRODUCTION}

$\mathbf{R}$ ECENTLY, the rapid development of solid-state lighting (SSL), especially high-brightness light-emitting diodes (LEDs), has attracted much attention. The advantages of LEDs include their high radiative efficiency, long lifetime, high tolerance to humidity, and limited heat generation [1], [2]. Moreover, the cost of LEDs, although still relatively high, decreases quickly due to the rapid development of semiconductor devices.

Manuscript received December 06, 2007; revised September 09, 2008. First published November 11, 2008; current version published February 13, 2009. The associate editor coordinating the review of this manuscript and approving it for publication was Prof. Brian L. Evans.

H. Yang is with the Department of Electrical Engineering, Eindhoven University of Technology, 5600 MB Eindhoven, The Netherlands. He is also with Philips Research Eindhoven, 5656 AE Eindhoven, The Netherlands (e-mail: h.m.yang@tue.nl).

J. W. M. Bergmans is with the Department of Electrical Engineering, Eindhoven University of Technology, The Netherlands (e-mail: j.w.m. bergmans@tue.nl).

T. C. W. Schenk and R. Rietman are with the Philips Research Eindhoven, 5656 AE Eindhoven, The Netherlands (e-mail: tim.schenk@philips.com; ronald.rietman@philips.com).

J.-P. M. G. Linnartz is with the Philips Research Eindhoven, 5656 AE Eindhoven, The Netherlands. He is also with the Department of Electrical Engineering, Eindhoven University of Technology, The Netherlands (e-mail: J.P. Linnartz@philips.com).

Color versions of one or more of the figures in this paper are available online at http://ieeexplore.ieee.org.

Digital Object Identifier 10.1109/TSP.2008.2009269
Because of these advantages, it is believed that LEDs will replace most of the conventional light sources in indoor illumination systems.

For the following reasons, it is beneficial to have a large number of spatially distributed LEDs in an indoor environment. First, the state-of-the-art LED technology [3] still cannot provide sufficient illumination by a single LED. Also, an extremely high-brightness LED luminary compromises eye safety. Moreover, spatially distributed LEDs can potentially provide localized illumination effects. By combining a large number of localized illumination effects, the LED based illumination system can offer more flexibility than conventional light sources, in rendering and changing the illuminance distributions [4]. Localized illumination effects can also reduce the light leakage into areas that do not need illumination so as to maintain energy conservation and prevent glare.

LEDs can be, in principle, arbitrarily distributed, however it is more convenient to have a regular array of LEDs on the ceiling, for the purpose of uniform lighting as well as product manufacturing. In this paper, we thus focus on the case with an array of LEDs in a regular grid which radiates light perpendicularly onto the floor. The considered scenario is illustrated schematically in Fig. 1. In an LED array, each single LED has a certain radiation pattern, and when fully switched on, the LED produces an illuminance distribution on the ground, called the basic illumination pattern, denoted as $f_{b}(x, y ; h)$, as also depicted schematically in Fig. 1. In this definition, the ground is viewed as an $(x, y)$ plane at a distance $h$ from the ceiling. When the LED is dimmed to some level, we also get a dimmed basic illumination pattern. The overall illuminance distribution due to multiple LEDs is a superposition of such basic illumination patterns each with potentially different illumination levels.

As introduced above, one of the important advantages of the LED illumination system is the ability to provide a localized illuminance distribution. The granularity of illumination rendering is closely related to the beam width of LEDs. Thus it is desirable to have as narrow a beam width as possible to achieve a fine granularity. However, the most important and common target illumination function in daily lives is still a uniform distribution around the room. Hence, in this paper, we discuss uniform illumination rendered by a regular array of LEDs with beams of prescribed width. In practice, the distance between the ground and the ceiling may not be known accurately. Moreover in certain cases, the distance may not be fixed or it is required to render a uniform illumination pattern at multiple distances from the ceiling simultaneously. Hence, in this paper, we consider uniform illumination rendering across a range of distances 


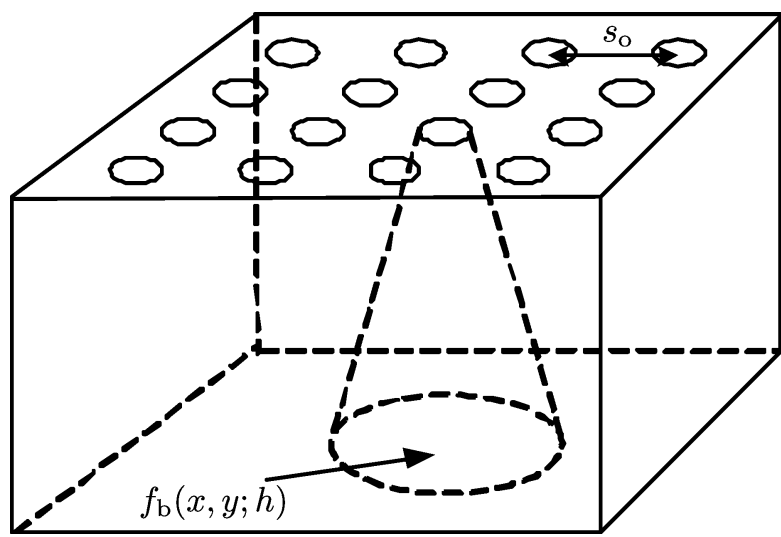

Fig. 1. A rectangular LED array applied for an indoor lighting system.

from the ceiling, or volumetric uniformity. In the literature, e.g., [4], [5], Moreno et al. worked on uniform illumination rendering by a finite LED array, and showed that the uniformity achieved changes with the distance from the ceiling and certain optimum distance can be computed for a given LED setting. In this paper, by comparison, we also investigate in detail the volumetric uniformity at every distance considered to show the evolution of uniformity achieved as the distance changes.

Further, in the literature, there are previous studies on uniform illumination rendering focusing on a finite number of LEDs [4]-[10], for which case the edge effects are significant. In this paper, by comparison, we do not consider the edge effects, because we are focusing on the center uniformity for the case when the number of LEDs is very large, e.g., thousands of LEDs in a room, and the area of a room is also very large, compared to the grid size of the LED array. In this case, the edge effects due to the limited number of LEDs are secondary. More specifically, we consider the limiting case with infinite number of LEDs in an infinitely large room.

For our scenario, we will first present two general results. The first states the scaling property of the basic illumination pattern $f_{b}(x, y ; h)$. We will show that the beam width is expanded, as the distance from the ceiling increases from $h_{1}$ to $h_{2}$, linearly by a ratio $h_{2} / h_{1}$. Hence considering illumination rendering, e.g., uniform illumination rendering, at different distances from the ceiling by the same LED array is equivalent to considering illumination rendering at one distance by LED arrays with different beam widths. The second general result claims that, for any $f_{b}(x, y ; h)$ and any grid shape for the LED array, the illumination pattern with maximum uniformity can be always achieved by setting the intensity levels of all the LEDs to be identical, as long as the cost function to measure uniformity is convex.

Given these two general results, in order to achieve an approximately uniform illumination effect, the remaining system parameters to be optimized are the basic illumination pattern $f_{b}(x, y ; h)$ and the grid shape of the LED array.

For the basic illumination pattern of each LED, we first illustrate its effect through some examples. As discussed above, we consider the illumination effects at different distances from the ceiling. In practice, a widely used model for the radiation pattern of LEDs is the generalized Lambertian function. In [11],
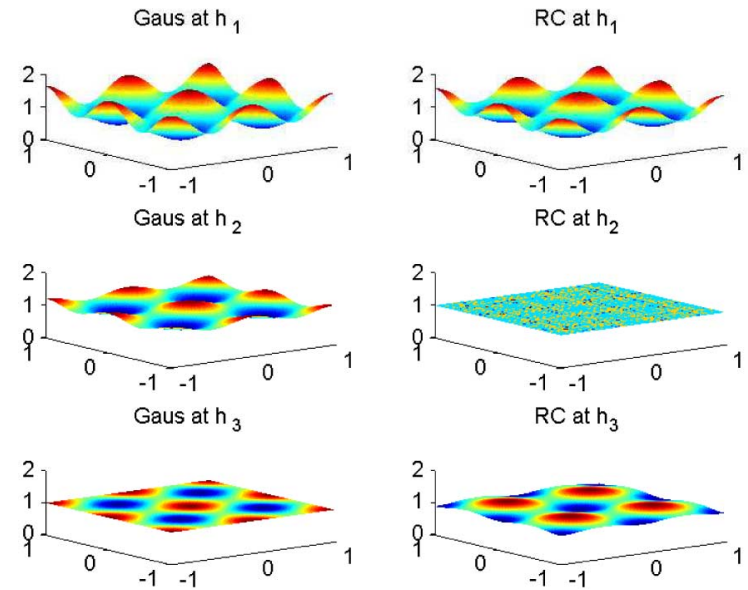

Fig. 2. Illumination pattern rendering at different distances from the ceiling, $h_{1}<h_{2}<h_{3}$. The $(x, y)$ coordinates denote the location of the illumination pattern, and the $z$ axis denotes the illuminance.

we show analytically that the basic illumination pattern due to the generalized Lambertian function can be well approximated by a Gaussian function defined mathematically in Section V-B. ${ }^{1}$ Hence, we first consider the Gaussian pattern in more detail. The rendered illumination pattern due to the Gaussian (Gaus) pattern by a rectangular array of LEDs is shown in the left column of Fig. 2, for multiple distances from the ceiling. It can be seen that the uniformity increases with the increase of the distance from the ceiling. This is, however, not true for all patterns. We present another example in the right column of Fig. 2, which is named the raised-cosine (RC) pattern, defined mathematically in Section V-C. The right column of Fig. 2 shows that the uniformity does not increase monotonically as the distance increases. From these examples, the design of $f_{b}(x, y ; h)$ appears to be a key issue, and is considered in more detail in later sections. We also propose an approach to optimize $f_{b}(x, y ; h)$ through a weighted combination of different known illumination patterns.

With respect to the grid shapes of the LED array, putting the LEDs in different regular grid shapes results in different performances in uniform illumination rendering. In this paper, we compare three regular grid shapes and show that significantly better uniformity can be achieved by using the hexagonal instead of the rectangular or triangular grids.

The rest of this paper is organized as follows. The two general results about the scaling property of $f_{b}(x, y ; h)$ and the setting of illumination levels of the LEDs are presented in Sections II and III, respectively. Key properties of human visual perception and the relative mean squared error (RMSE) function are introduced in Section IV. We compare the performances of different basic patterns and the linear combinations of some of these patterns in Section V. The performances of uniform illumination rendered by an LED array with triangular, rectangular, and hexagonal grid shapes are compared in Section VI. Finally, Section VII concludes this paper.

${ }^{1}$ Note that the Gaussian pattern here is defined as a function of $(x, y ; h)$. In the literature, e.g., [8], [9], there is a different definition for the Gaussian pattern as a function of the observation angle. 


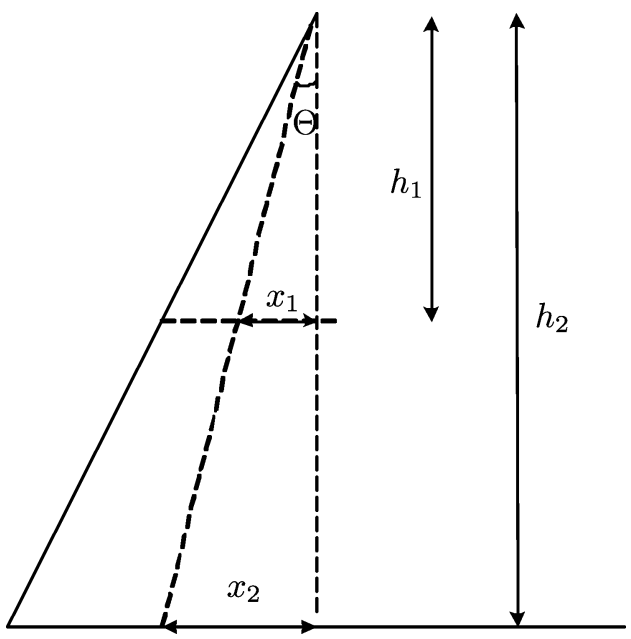

Fig. 3. One dimensional illustration of the geometry for the illumination effect at different distances $h$ from the ceiling, where $\Theta$ denotes the solid angle.

\section{SCALING Property OF the BASIC Illumination PATtERn AND ILLUMINATION RENDERING}

For illumination rendering, each LED can be viewed as a point light source and therefore the radiation pattern of each LED can be viewed as a function of the solid angle $f(\Theta)$ in the three-dimensional space. When $f(\Theta)$ is mapped onto a flat surface at certain distance, denoted by $h$, from the ceiling, we obtain the basic illumination pattern $f_{b}(x, y ; h)$. More specifically, we have $f_{b}(x, y ; h)=\left(f(\Theta) / x^{2}+y^{2}+h^{2}\right)$ due to the definition of the solid angle [12].

Considering the basic illumination patterns at two different distances $h_{1}$ and $h_{2}$, as depicted in Fig. 3, we obtain that $f_{b}\left(x_{1}, y_{1} ; h_{1}\right)=f(\Theta) /\left(h_{1}^{2}+x_{1}^{2}+y_{1}^{2}\right)$ and $f_{b}\left(x_{2}, y_{2} ; h_{2}\right)=f(\Theta) /\left(h_{2}^{2}+x_{2}^{2}+y_{2}^{2}\right)$. Letting $r_{h} \triangleq h_{2} / h_{1}=x_{2} / x_{1}=y_{2} / y_{1}$, we get $f_{b}\left(x_{2}, y_{2} ; h_{2}\right)=$ $f_{b}\left(x_{1}, y_{1} ; h_{1}\right) / r_{h}^{2}$, i.e.

$$
f_{b}\left(x, y ; r_{h} h\right)=\frac{1}{r_{h}^{2}} f_{b}\left(\frac{x}{r_{h}}, \frac{y}{r_{h}} ; h\right) .
$$

It can be seen from (1) that the basic illumination pattern at $r_{h} h$ is identical to that at $h$ with its beam width scaled by $r_{h}$. Of course the total light energy does not scale with $h$, as can be obtained from (1)

$$
\int_{-\infty}^{\infty} \int_{-\infty}^{\infty} f_{b}\left(x, y ; r_{h} h\right) \mathrm{d} x \mathrm{~d} y=\int_{-\infty}^{\infty} \int_{-\infty}^{\infty} f_{b}(x, y ; h) \mathrm{d} x \mathrm{~d} y .
$$

Moreover, the overall illumination pattern is a linear superposition of the basic illumination patterns from multiple LEDs. Let $f_{v}(x, y ; h, \omega)$ denote the overall illumination pattern at $h$ implemented by an LED array with the beam width of each LED to be $\omega$, i.e.

$$
f_{v}(x, y ; h, \omega)=\sum_{i} \frac{v_{i}}{\omega^{2}} f_{b}\left(\frac{x-x_{i}}{\omega}, \frac{y-y_{i}}{\omega} ; h\right)
$$

where $\left(x_{i}, y_{i}\right)$ and $v_{i}$ represent the location and intensity level of the $i$ th LED, respectively, and $0 \leq v_{i} \leq 1$. Note that the specific definition for beam width is irrelevant to (2), and discussions on the definition of will be provided Section V-D. From (1) and (2), we can obtain the following property.
1) Property 1: Scaling property of illumination rendering

The illumination, denoted by $f_{v}(x, y ; h, \omega)$, at distance $h$ from the ceiling, realized by an LED array with the beam width of each LED to be $\omega$, satisfies

$$
f_{v}\left(x, y ; r_{h} h, \omega\right)=f_{v}\left(x, y ; h, r_{h} \omega\right)
$$

i.e., the scaling of $h$ and $\omega$ are interchangeable when considering illumination rendering by an LED array.

From Property 1, we will only consider illumination rendering over a range of distances from the ceiling by an LED array with fixed beam width in the rest of the paper. The results obtained can be easily extended into the case when the beam width of the LEDs can be also adjusted. Hence in Sections III-VI, the parameter $\omega$ will be dropped when writing relevant functions.

\section{OPTIMUM INTENSITY LEVEL SETTING FOR UNIFORM ILLUMINATION RENDERING}

In order to produce a uniform illumination pattern, we set LEDs to some illumination levels, $\left\{v_{i}\right\}$. We define an error function, $f_{e}(x, y ; h) \triangleq f_{v}(x, y ; h)-f_{t}$, where $f_{t}$ is the target uniform illumination pattern which is constant over the $(x, y)$ space. A cost function, denoted by $\xi\left(f_{e}\right)$, can thus be used to measure the level of uniformity achieved. An optimum LED intensity setting, $\left\{v_{i, o}\right\}$, is the setting such that $\xi\left(f_{e}\right)$ is minimized. A large category of possible cost functions, such as $\xi\left(f_{e}\right)=\max _{x, y}\left|f_{e}(x, y)\right|$ and $\xi\left(f_{e}\right)=\int_{x} \int_{y} f_{e}^{2}(x, y) \mathrm{d} x \mathrm{~d} y$, can be shown to be convex functions with respect to $f_{e}$, i.e.

$$
\xi\left(\alpha f_{e, 1}+(1-\alpha) f_{e, 2}\right) \leq \alpha \xi\left(f_{e, 1}\right)+(1-\alpha) \xi\left(f_{e, 2}\right)
$$

where $f_{e, 1}$ and $f_{e, 2}$ are two error functions due to different setting $\left\{v_{i}\right\}$, and $0 \leq \alpha \leq 1$.

For such commonly used convex cost functions, and due to the shift-invariance of the rendered illumination pattern with respect to a regular LED grid, we can obtain the optimum LED intensity level setting as follows.

First consider an arbitrary setting of the LED intensity levels and denote it as a vector $\boldsymbol{v}_{1} \triangleq\left[v_{1}, v_{2}, \ldots\right]^{T}$, where $(\cdot)^{T}$ denotes the transpose operation. We can simply shift the LED illumination level settings by one LED with respect to the LED array, e.g., the new illumination level of each LED equals the original level of the LED next to it on the left. ${ }^{2}$ Let $\boldsymbol{v}_{2}$ denote the new LED intensity levels. It is clear that the illumination effect is shift-invariant under the scenario considered in this paper, i.e.

$$
\xi\left(f_{e} \mid \boldsymbol{v}_{1}\right)=\xi\left(f_{e} \mid \boldsymbol{v}_{2}\right) .
$$

${ }^{2}$ As an example to illustrate this operation, we look at one infinite row of LEDs with illumination levels to be

$$
\ldots, v_{0}, v_{1}, v_{2}, v_{0}, v_{1}, v_{2} \ldots
$$

corresponding to the LED locations. Now, we can compose another setting to be

$$
\ldots, v_{2}, v_{0}, v_{1}, v_{2}, v_{0}, v_{1} \ldots
$$

while the locations of LEDs do not change. These two settings will give the identical performance in terms of uniform illumination rendering. Note that we give an example of repetitive setting for illustration, this is however not necessary to prove Property 2. 
We then obtain the average of $\boldsymbol{v}_{1}$ and $\boldsymbol{v}_{2}$, i.e., $\boldsymbol{v}_{\text {ave }}=\left(\boldsymbol{v}_{1}+\right.$ $\left.\boldsymbol{v}_{2}\right) / 2$. It is clear that the average illuminance rendered due to $\boldsymbol{v}_{1}, \boldsymbol{v}_{2}$ and $\boldsymbol{v}_{\text {ave }}$ are all the same. Furthermore, we have that $f_{e} \mid \boldsymbol{v}_{\text {ave }}=\left(f_{e}\left|\boldsymbol{v}_{1}+f_{e}\right| \boldsymbol{v}_{2}\right) / 2$. Due to the convexity of the cost function, we have

$$
\xi\left(f_{e} \mid \boldsymbol{v}_{\text {ave }}\right) \leq \frac{1}{2}\left(\xi\left(f_{e} \mid \boldsymbol{v}_{1}\right)+\xi\left(f_{e} \mid \boldsymbol{v}_{2}\right)\right)=\xi\left(f_{e} \mid \boldsymbol{v}_{1}\right)
$$

where the last equality holds because of (5). Therefore, the level of uniformity in the illumination effect achieved by any LED illumination level, $\boldsymbol{v}_{1}$, is upper-bounded by that achieved by a corresponding shifted-and-averaged level $\boldsymbol{v}_{\text {ave }}$.

The above shift-and-average operation can be further extended to arbitrary number of shifts, denoted by $N_{\text {shift }}$, with respect to the LED array, i.e., $\boldsymbol{v}_{\text {ave }}=\left(1 / N_{\text {shift }}\right) \sum_{i=1}^{N_{\text {shift }}} \boldsymbol{v}_{\boldsymbol{i}}$. When $N_{\text {shift }}$ gets large, $\boldsymbol{v}_{\text {ave }}$ asymptotically becomes the vector where all the elements are identical, i.e., a uniform setting of intensity levels. Further, if $\boldsymbol{v}_{1}$ is a uniform illumination setting, $\boldsymbol{v}_{\text {ave }}$ is equal to $\boldsymbol{v}_{1}$, and thus the cost function can not be further minimized by this shift-and-average operation. Hence we obtain the following property.

1) Property 2: If the cost function to measure the uniformity in terms of illumination rendering, $\xi\left(f_{e}\right)$, is convex with respect to $f_{e}$, and due to the shift-invariance property of a rendered illumination pattern, the optimum uniformity can be achieved by setting the LED intensity levels to be identical.

Note that if the given convex cost functions are minimized by the setting $\boldsymbol{v}_{\text {ave }}$, any monotonic function of such convex functions will be also minimized, such as $\xi\left(f_{e}\right)=$ $\log \max _{x, y}\left|f_{e}(x, y)\right|$ and $\xi\left(f_{e}\right)=\sqrt{\int_{x} \int_{y} f_{e}^{2}(x, y) \mathrm{d} x \mathrm{~d} y}$.

Finally, note that the general result presented above in this section is focused on the case without considering the edge effects, as discussed in the Section I. If the number of LEDs is small, the edge effects can be quite visible, thus different intensity level setting can be used. For instance, a viable option is to set the intensity levels at the edge of the LED array differently from that in the center of the LED array [4], [5].

\section{Human Perception AND COST FunCtion}

As aforementioned, some cost function needs to be used to measure the uniformity achieved in terms of illumination rendering. In this section, we discuss a few properties of human perception and propose a convex cost function based on RMSE. Similar MSE based cost functions were also used in [7], [9].

\section{A. Human Perception}

When looking at an illuminated target location, the human visual system treats it as a light source and the exact measurement of the light intensity perceived, called luminance, is the amount of light reflected from the location per unit area in the direction of the eyes. This measurement is dependent on many factors, e.g., the type of the reflecting surface and the position of the eyes. Therefore a widely used criterion for designing illumination effects is the measurement of the illumination intensity incident on the target location, also called illuminance [13], measured in lumen $/ \mathrm{m}^{2}$.

The human visual system in principle can process a very wide range of illuminance, however not all at the same time.
It continuously adapts itself according to the background or environmental lighting conditions. In general, the human visual system is more sensitive to the light in a dark background than in a bright background. For uniform illumination rendering, the target illumination effect is a uniform background lighting. Therefore, the "visibility" of an illumination distortion or illumination pattern at the target location can be characterized by the contrast ratio $C=f_{e}(x, y ; h) / f_{t}$ [13]. This characterization is also consistent with the Weber's law for human perception [13]. Further, there is in practice a threshold for $C$, denoted by $C_{\text {th }}$, below which $f_{e}(x, y ; h)$ is not visible.

\section{B. Cost Function}

In this section, we propose a MSE based cost function for analytical tractability, taking human perception properties into consideration. More specifically, we propose RMSE as the cost function, i.e.

$$
\xi_{\text {RMSE }}=\sqrt{\frac{1}{\|\mathcal{A}\|} \int_{(x, y ; h) \in \mathcal{A}} \frac{f_{e}^{2}(x, y ; h)}{f_{t}^{2}} \mathrm{~d}(x, y ; h)}
$$

where $\mathcal{A}$ is the region of the illumination pattern considered. To investigate uniform illumination in an infinite space by a regular LED array, we later show that we only need to compute the RMSE in one or a few, depending on the LED grid shapes, unit areas corresponding to an LED grid. The RMSE is normally shown in $\mathrm{dB}$ scale, i.e.

$$
\xi_{\mathrm{RMSE}}(\mathrm{dB})=10 \log _{10} \xi_{\mathrm{RMSE}} .
$$

Next, we give a quantitative example of the range of MSEs that is perceivable based on the threshold properties introduced in Section IV-A. Assume by way of illustration that there is a two-dimensional raised-cosine illumination pattern, the exact definition of which is shown in Section V-C, on a uniform lighting background at the distance $h$ from the ceiling, and that the contrast ratio between the illuminance of the raised-cosine illumination pattern and that of the background is exactly $C_{\mathrm{th}}$. More precisely, assume that

$$
\frac{f_{e}(x, y ; h)}{f_{t}}=C_{\mathrm{th}}\left(1+\cos \frac{2 \pi}{s_{o}} x\right)\left(1+\cos \frac{2 \pi}{s_{o}} y\right)
$$

where $s_{o}$ denotes the distance between neighboring LEDs on the ceiling. For this case, $\mathcal{A}$ in (7) is the region where $-s_{o} / 2 \leq$ $x, y \leq s_{o} / 2$. This example is used since it represents the key spatial frequency component in the distortion for uniform illumination rendering by a square array of LEDs with grid size $s_{o}$, as illustrated in Fig. 1. By comparing (7) and (9), we obtain that $\xi_{\mathrm{RMSE}}=(3 / 2) C_{\mathrm{th}}$. From [13], it is shown that $C_{\mathrm{th}}$ may vary from 0.01 to 100 depending on many factors, such as the background illumination level, i.e., $f_{t}$ in this paper, and direction of the observer. For instance, $C_{\text {th }}$ is larger when $f_{t}$ is higher. Hence, the perceivable $\xi_{\mathrm{RMSE}}$ for an observer can range from -18 to $22 \mathrm{~dB}$ in practice. Hence, RMSE values well outside this range are of no interest to this paper.

Given the definition of the RMSE cost function, we are ready to evaluate the performance of uniform illumination rendering across a range of distances from the ceiling. Based on the evaluation, we can also optimize system parameters to achieve more 
uniform illumination patterns. From Property 1 and Property 2, it is clear that, in order to achieve uniform illumination, the remaining system parameters to be optimized are the basic illumination pattern $f_{b}(x, y ; h)$ and the grid shape of the LED array, which will be discussed in Sections V and VI, respectively.

\section{Design OF BASIC Illumination PATtern}

In this section, we investigate the uniformity in the illumination effect achieved by the uniform LED illumination level setting for a rectangular array of LEDs, in order to compare and design basic illumination patterns $f_{b}(x, y ; h)$. In this case, the $\left(x_{i}, y_{i}\right)$ locations of the LEDs can be written as $\left(l s_{x}, n s_{y}\right)$, where both $l$ and $n$ are integers. Without loss of generality, we first consider the square grid, i.e., $s_{x}=s_{y}=s_{o}$, in Section V-A. Further reason about why the square grid is of particular interest is discussed in Section VI. Extensions of the results obtained for the square grid into general rectangular grid is trivial and thus not shown in this paper. Further extension of the results obtained for the rectangular grid to other regular grid shapes will also be presented in Section VI.

\section{A. Evaluation of RMSE}

First, we derive the exact expression of RMSE when the LED intensity levels are set to be identical according to Property 2 .

Let $v_{c}$ denote the illumination levels of the LEDs, where $v_{c}$ is a constant according to Property 2 . The target illumination effect $f_{t}$ is also the mean value of the illuminance on a flat surface at distance $h$ that can be evaluated as follows:

$$
\begin{aligned}
f_{t} & =\frac{1}{s_{o}^{2}} \int_{-s_{o} / 2}^{s_{o} / 2} \int_{-s_{o} / 2}^{s_{o} / 2} \sum_{l=-\infty}^{\infty} \sum_{n=-\infty}^{\infty} v_{c} f_{b}\left(x-l s_{o}, y-n s_{o} ; h\right) \mathrm{d} x \mathrm{~d} y \\
& =\sum_{l=-\infty}^{\infty} \sum_{n=-\infty}^{\infty} \frac{1}{s_{o}^{2}} \int_{(l-(1 / 2)) s_{o}}^{(l+(1 / 2)) s_{o}} \int_{(n-(1 / 2)) s_{o}}^{(n+(1 / 2)) s_{o}} v_{c} f_{b}(x, y ; h) \mathrm{d} x \mathrm{~d} y \\
& =\frac{v_{c}}{s_{o}^{2}} \int_{-\infty}^{\infty} \int_{-\infty}^{\infty} f_{b}(x, y ; h) \mathrm{d} x \mathrm{~d} y \\
& =\frac{v_{c} P_{b}}{s_{o}^{2}}
\end{aligned}
$$

where $P_{b}$ is the total flux of an LED, i.e., $P_{b}=$ $\int_{-\infty}^{\infty} \int_{-\infty}^{\infty} f_{b}(x, y ; h) \mathrm{d} x \mathrm{~d} y$. Intuitively, (10) can be explained as follows. Due to the symmetry of the LED array and the uniform illumination level setting, the optical power shed on to each $s_{O} \times s_{O}$ area around the $(x, y)$ location of an LED is identical. The total power shed on the surface from each LED is $v_{c} P_{b}$. Hence, the mean value of illuminance is given by

$$
f_{t}=\lim _{N \rightarrow \infty} \frac{N v_{c} P_{b}}{N s_{o}^{2}}=\frac{v_{c} P_{b}}{s_{o}^{2}} .
$$

We can thus further evaluate the performance of rendering such a uniform illumination pattern through considering the RMSE cost function defined in (7), yielding (12), as shown at the bottom of the page. In (12), $F_{b}(u, v ; h)$ is the Fourier transform of $f_{b}(x, y ; h)$, known as

$$
\begin{aligned}
F_{b}(u, v ; h) \triangleq \int_{-\infty}^{\infty} \int_{-\infty}^{\infty} & f_{b}(x, y ; h) \\
& \times \exp (-2 \pi j(u x+v y)) \mathrm{d} x \mathrm{~d} y .
\end{aligned}
$$

A detailed derivation of (12) is shown in Appendix I. Effectively, $\xi_{\text {RMSE }}$ is obtained through sampling $F_{b}$ and the summation of all the samples except the DC component.

Until now, we only discussed the light shed on the surface directly from LEDs. Light may also propagate through one or more diffuse reflections and eventually arrive at some location. Due to the nature of diffuse reflections, the light from diffuse reflections is approximately uniformly distributed [14]. There may exist other light sources, e.g., fluorescent lamps, in an indoor environment, the illuminance due to which can be also viewed as uniformly distributed. In the presence of uniform background light, the absolute MSE in terms of rendering uniform illumination does not change. The effect on the RMSE can be fully accounted for by incorporating the power from diffuse reflections and background light into $f_{t}$, and modifying it into $\hat{f}_{t}=\gamma f_{t}$. Therefore, we get $\hat{\xi}_{\mathrm{RMSE}}(h)=(1 / \gamma) \xi_{\mathrm{RMSE}}(h)$. The ratio $\gamma$ is close to one when there is no strong background light. As a numerical example, for the ceiling and floor with $50 \%$ reflectivity, $\gamma$ is about 1.33 , when considering infinite number of reflections. In this case, $\xi_{\mathrm{RMSE}}$ is only $1.24 \mathrm{~dB}$ lower due to the reflected light. We will also later see that this $\gamma$ does not influence the main conclusions of this paper. In the rest of this paper, we focus on the case without strong background light, and, hence, $\gamma$ is neglected.

From (12), $\xi_{\mathrm{RMSE}}(h)$ remains a function of $h$, and (12) in fact presents a convenient approach to compute $\xi_{\mathrm{RMSE}}(h) \mathrm{nu}-$ merically, in comparison to the original two-dimensional integral which is quite complicated to compute. Furthermore, from (1), we can obtain that

$$
F_{b}\left(u, v ; r_{h} h\right)=F_{b}\left(r_{h} u, r_{h} v ; h\right) .
$$

$$
\begin{aligned}
\xi_{\mathrm{RMSE}}(h) & =\left[\frac{1}{f_{t}^{2}} \frac{1}{s_{o}^{2}} \int_{-s_{o} / 2}^{s_{o} / 2} \int_{-s_{o} / 2}^{s_{o} / 2}\left(\sum_{l=-\infty}^{\infty} \sum_{n=-\infty}^{\infty} v_{c} f_{b}\left(x-l s_{o}, y-n s_{o} ; h\right)-f_{t}\right)^{2} \mathrm{~d} x \mathrm{~d} y\right]^{1 / 2} \\
& =\frac{1}{P_{b}} \sqrt{\sum_{\left\{l, n \mid l^{2}+n^{2} \neq 0\right\}}\left|F_{b}\left(l \frac{1}{s_{o}}, n \frac{1}{s_{o}} ; h\right)\right|^{2}}
\end{aligned}
$$


Hence from (12), we get

$$
\begin{aligned}
& \xi_{\mathrm{RMSE}}\left(r_{h} h\right) \\
& =\frac{1}{P_{b}} \sqrt{\sum_{\left\{l, n \mid l^{2}+n^{2} \neq 0\right\}}\left|F_{b}\left(l \frac{1}{s_{O}}, n \frac{1}{s_{O}} ; r_{h} h\right)\right|^{2}} \\
& =\frac{1}{P_{b}} \sqrt{\sum_{\left\{l, n \mid l^{2}+n^{2} \neq 0\right\}}\left|F_{b}\left(l \frac{r_{h}}{s_{O}}, n \frac{r_{h}}{s_{O}} ; h\right)\right|^{2}} .
\end{aligned}
$$

This expression shows that, as the distance $h$ changes to $r_{h} h$, $\xi_{\text {RMSE }}$ can be simply obtained by changing the sampling rate of $F_{b}(u, v ; h)$ from $1 / s_{o}$ into $r_{h} / s_{o}$. Thus, (15) further gives a low-complexity approach to evaluate $\xi_{\mathrm{RMSE}}$ across a range of distances from the ceiling.

Now, it is shown that $\xi_{\mathrm{RMSE}}$ is determined by the function $F_{b}(u, v ; h)$ or $f_{b}(x, y ; h)$. Further, from $(15)$, we are ready to design $f_{b}(x, y ; h)$ in order to achieve minimal $\xi_{\mathrm{RMSE}}$. Before that, we first compare the performances of a few known functions as $f_{b}(x, y ; h)$.

\section{B. Performance of a Gaussian Basic Illumination Pattern}

In practice, a widely used model for the radiation pattern of LEDs is the generalized Lambertian function. In [11], we show that $f_{b}(x, y ; h)$ in this case can be well approximated by a twodimensional Gaussian function $f_{g}(x, y ; h)$, i.e.

$$
f_{g}(x, y ; h)=\frac{c_{h}}{2 \pi \sigma_{h}^{2}} \exp \left\{-\frac{x^{2}+y^{2}}{2 \sigma_{h}^{2}}\right\}
$$

where $\sigma_{h}^{2}$ is the variance and $c_{h}$ is a normalization factor. The Fourier transform of $f_{g}(x, y ; h)$ can be obtained as $F_{g}(u, v ; h)=c_{h} \exp \left(-2 \pi^{2} \sigma_{h}^{2}\left(u^{2}+v^{2}\right)\right)$.

The RMSE function in (12) can be derived as follows:

$$
\begin{aligned}
\xi_{\mathrm{RMSE}}(h) & =\frac{1}{P_{b}} \sqrt{\sum_{\left\{l, n \mid l^{2}+n^{2} \neq 0\right\}}\left|F_{g}\left(l \frac{1}{s_{o}}, n \frac{1}{s_{o}} ; h\right)\right|^{2}} \\
& =\frac{c_{h}}{P_{b}} \sqrt{\left(\vartheta_{3}\left(0, \exp \left(-\frac{4 \pi^{2} \sigma_{h}^{2}}{s_{o}^{2}}\right)\right)\right)^{2}-1}
\end{aligned}
$$

where $\vartheta_{3}(\cdot, \cdot)$ is the Jacobi theta function of type three. Numerical results for $\xi_{\mathrm{RMSE}}(h)$ are given in Fig. 7.

From (15) and the following Lemma 1 , it can be seen that $\xi_{\mathrm{RMSE}}(h)$ is monotonically decreasing as $h$ increases, as was illustrated in Fig. 2.

Lemma 1: A sufficient condition for $\xi_{\mathrm{RMSE}}$ to be monotonically decreasing as $h$ increases is that $\left|F_{b}(u, v)\right|^{2}$ is also monotonically decreasing as $|u|$ or $|v|$ increases.
Proof: From (15), we have (18), as shown at the bottom of the page. Since $\partial\left(\left|F_{b}(u, v ; h)\right|^{2}\right) / \partial u \leq 0$ when $u \geq 0$ and $\partial\left(\left|F_{b}(u, v ; h)\right|^{2}\right) / \partial u \geq 0$ when $u \leq 0$, and since the same property applies to the partial derivative of $\left|F_{b}(u, v ; h)\right|^{2}$ by $v$, we have $\mathrm{d}\left(\xi_{\mathrm{RMSE}}^{2}\left(r_{h} h\right)\right) / \mathrm{d}\left(r_{h}\right) \leq 0$. Because $\mathrm{d}\left(\xi_{\mathrm{RMSE}}^{2}\left(r_{h} h\right)\right) /$ $\mathrm{d}\left(r_{h}\right)=2 \xi_{\mathrm{RMSE}}\left(r_{h} h\right) \mathrm{d}\left(\xi_{\mathrm{RMSE}}\left(r_{h} h\right)\right) / \mathrm{d}\left(r_{h}\right)$, and $\xi_{\mathrm{RMSE}}\left(r_{h} h\right) \geq 0$, we have $\mathrm{d}\left(\xi_{\mathrm{RMSE}}\left(r_{h} h\right)\right) / \mathrm{d}\left(r_{h}\right) \leq 0$.

From Lemma 1, we can identify many other functions $f_{b}(x, y ; h)$ as basic illumination patterns, for which $\xi_{\mathrm{RMSE}}(h)$ keeps decreasing as $h$ increases. This is a useful property in the sense that we only need to design the LED patterns to satisfy some requirement on $\xi_{\mathrm{RMSE}}(h)$ at a certain target distance $h_{0}$ and do not need to pay attention to $\xi_{\mathrm{RMSE}}$ at a larger distance. Examples of such functions include the linear roll-off function, $f_{b}(x, y)=c_{1}\left(1-c_{2}|x|\right)\left(1-c_{3}|y|\right)$, the two-dimensional Gaussian function, the exponential function $f_{b}(x, y)=c_{1} \exp \left(-c_{2}|x|-c_{3}|y|\right)$, and the Lorenzian function $f_{b}(x, y) \propto\left[\left(c_{1}^{2}+x^{2}\right)\left(c_{2}^{2}+y^{2}\right)\right]^{-1}$, where $c_{1}, c_{2}$ and $c_{3}$ are constants.

Although the Gaussian basic pattern and some other patterns introduced by now have the appealing property that the resulting $\xi_{\mathrm{RMSE}}(h)$ is monotonic, perfect uniformity, i.e., $\xi_{\mathrm{RMSE}}(h)=$ 0 , can never be achieved at any distance $h$. In Section V-C, by comparison, we are going to present a family of basic illumination patterns that will make $\xi_{\mathrm{RMSE}}(h)=0$ at certain distances.

\section{Performances of the Family of Raised-Cosine Patterns}

From (12), $\xi_{\mathrm{RMSE}}=0$ at certain $h_{0}$, if and only if $F_{b}\left(l / s_{o}, n / s_{o} ; h_{0}\right)$ is nonzero when $n=0$ and $l=0$, and zero otherwise. Further, after working out the scaling parameters, we have an extension of the well known Nyquist pulse shaping criterion [15].

Lemma 2: Extended Nyquist Pulse Shaping Criterion:

The necessary and sufficient condition for

$$
\sum_{l=-\infty}^{\infty} \sum_{n=-\infty}^{\infty} f_{b}\left(x-l s_{o}, y-n s_{o} ; h_{0}\right)=1, \quad \forall(x, y)
$$

is

$$
F_{b}\left(l \frac{1}{s_{o}}, n \frac{1}{s_{o}} ; h_{0}\right)=s_{o}^{2} \delta(l, n)
$$

where $\delta(\cdot)$ is the Kronecker delta function. Lemma 2 defines an entire class of functions $f_{b}(x, y ; h)$, through which we can achieve perfect uniformity at $h_{0}$. Of particular interest in this paper, we consider the family of separable raised-cosine functions due to two reasons. One is that it is limited in the spatial domain. The other is that this family of functions

$$
\frac{\mathrm{d} \xi_{\mathrm{RMSE}}^{2}\left(r_{h} h\right)}{\mathrm{d} r_{h}}=\frac{1}{P_{b}^{2}} \sum_{\left\{l, n \mid l^{2}+n^{2} \neq 0\right\}}\left(\left.\frac{\partial\left|F_{b}(u, v ; h)\right|^{2}}{\partial u}\right|_{u=l\left(r_{h} / s_{o}\right)} \cdot \frac{l}{s_{O}}+\left.\frac{\partial\left|F_{b}(u, v ; h)\right|^{2}}{\partial v}\right|_{v=n\left(r_{h} / s_{o}\right)} \cdot \frac{n}{s_{o}}\right) .
$$


includes shapes with different steepness corresponding to different roll-off factor $\beta$. The raised-cosine function at $h_{0}$ can be written as

$$
\begin{aligned}
& f_{b}\left(x, y ; h_{0}\right)=\operatorname{rcos}(x ; \beta) \operatorname{rcos}(y ; \beta) \\
& F_{b}\left(u, v ; h_{0}\right)=\operatorname{Frcos}(u ; \beta) \operatorname{Frcos}(v ; \beta)
\end{aligned}
$$

where

$$
\begin{aligned}
& \operatorname{rcos}(x ; \beta) \\
& \quad= \begin{cases}1, & |x| \leq \frac{1-\beta}{2} s_{o} \\
\frac{1}{2}+\frac{1}{2} \cos \left(\frac{\pi}{\beta s_{o}}\left(|x|-\frac{1-\beta}{2} s_{o}\right)\right), & \frac{1-\beta}{2} s_{o}<|x| \leq \frac{1+\beta}{2} s_{o} \\
0, & \text { elsewhere }\end{cases}
\end{aligned}
$$

and its Fourier transform

$$
\operatorname{Frcos}(u ; \beta)=s_{o} \operatorname{sinc}\left(\pi u s_{o}\right) \frac{\cos \left(\pi \beta u s_{o}\right)}{1-4 \beta^{2} u^{2} s_{o}^{2}} .
$$

Note that the expressions for the raised-cosine pattern, (21) to (24), at $h_{0}$ do not depend on the value of $h_{0}$, while only depend on $s_{0}$. A practical example for such raised-cosine pattern can be $h_{0}=2 \mathrm{~m}$ while $s_{o}=0.1 \mathrm{~m}$, for an LED array with narrow beams. The general expression at any $h$ for $f_{b}(x, y ; h)$ and $F_{b}\left(u, v ; r_{h} h\right)$ can be obtained through (1) and (14).

For this particular family of basic patterns, perfect uniformity is achieved at $h_{o}$. At this distance, the $-3 \mathrm{~dB}$ beam width is $s_{o} / 2$, i.e., the value of $f_{b}(x, y ; h)$ decreases to half of its maximum at the middle of two neighboring LEDs. More discussions on beam width definitions are presented in Section V-D. The illumination pattern rendered at another distance $h$ can be expressed as a function of $h / h_{o}$.

For $h / h_{o}$ smaller than or greater than one, $\xi_{\mathrm{RMSE}}(h)$ becomes nonzero. When $h / h_{o}$ decreases below one, $\xi_{\mathrm{RMSE}}(h)$ keeps increasing. On the contrary, as $h / h_{o}$ increases above one, $\xi_{\mathrm{RMSE}}$ will be reduced again. After many fluctuations, $\xi_{\mathrm{RMSE}}$ approaches zero as $h / h_{o}$ goes to infinity. Further for the class of raised-cosine functions, we focus on the case when $h_{o}$ corresponds to the first null of $F_{b}(u, v ; h)$ to provide the lowest possible beam width, and thus the minimum illumination granularity can be achieved.

Fig. 4 illustrates the trend of $\xi_{\mathrm{RMSE}}(h)$ as $h$ changes, for raised-cosine functions with different roll-off factors, denoted by $\beta$. Note that it is in general difficult to obtain closed-form expressions for (15), however, for the functions we are interested in this paper, e.g., raised-cosine functions, the dominant factors in (15) are only the first few terms. It may be shown through numerical computations that (15) almost converges as $l$ and $n$ are summed until 20.

It can be seen from Fig. 4 that, although a raised-cosine function $f_{b}$ with any roll-off factor $\beta$ results in perfect uniformity at $h_{o}, \xi_{\mathrm{RMSE}}$ at other distances can be quite different at different $\beta$. In general, $\xi_{\mathrm{RMSE}}$ is lower at a higher $\beta$. More specifically, we compare the worst case $\xi_{\mathrm{RMSE}}$, i.e., the largest $\xi_{\mathrm{RMSE}}$, in the range $h \geq h_{o}$ and depict the results in Fig. 5. It is seen that the

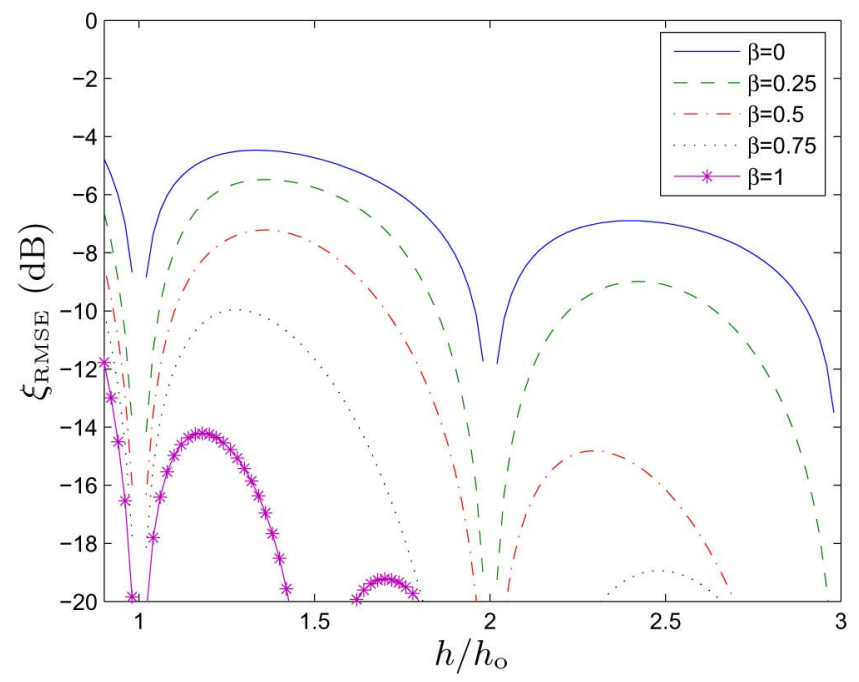

Fig. 4. The cost function $\xi_{\mathrm{RMSE}}$ versus the normalized distance $h / h_{o}$.

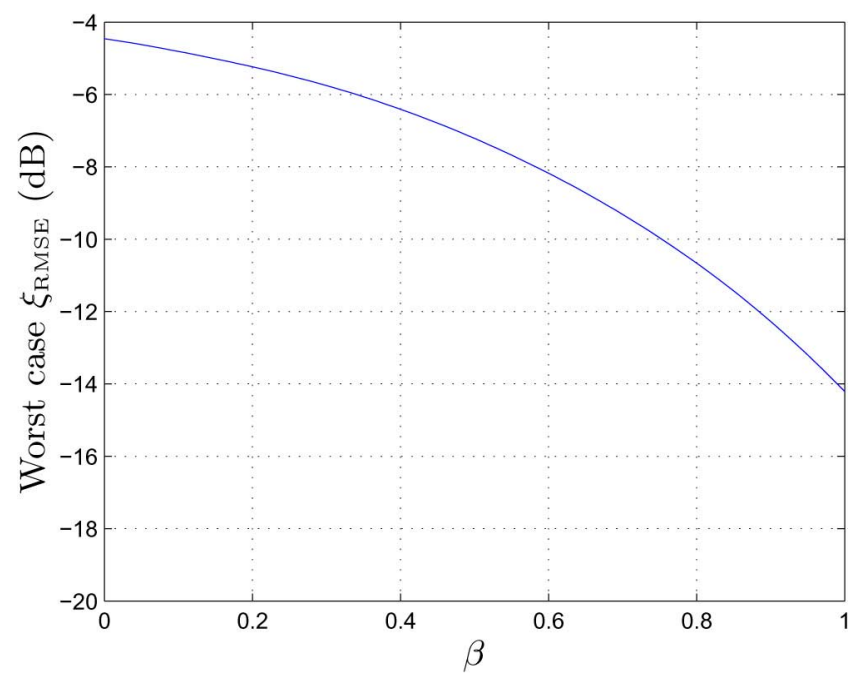

Fig. 5. Worst case $\xi_{\mathrm{RMSE}}$ versus roll-off factor $\beta$.

raised-cosine function with $\beta=1$ outperforms all other functions with smaller $\beta$. Hence in the rest of this paper, we only focus on the case with $\beta=1$.

In this section, we discussed the optimum $f_{b}$ according to the extended Nyquist pulse shaping criterion. The applicability of such non-Gaussian basic patterns is due to the assumption that we are allowed to implement such $f_{b}$ through designing the optical component attached to the LEDs. Some practical constraints on $f_{b}$ considered in this paper include $f_{b}>0, f_{b}$ is symmetrical, i.e., $f_{b}(-x, y)=f_{b}(x, y)$ and $f_{b}(x,-y)=f_{b}(x, y)$, or rotationally symmetrical, i.e., $f_{b}(x, y)=f_{b}(x \cos \phi-y \sin \phi, y \cos \phi+x \sin \phi), \forall \phi \in[0,2 \pi]$, in the spatial domain for convenience in manufacturing. Moreover, $f_{b}$ is monotonically decreasing from the center of the beam.

\section{Comparison of Basic Patterns $f_{b}$}

In this section, we compare different basic illumination patterns based on their performances in uniform illumination rendering across a range of distances. To compare the performance 


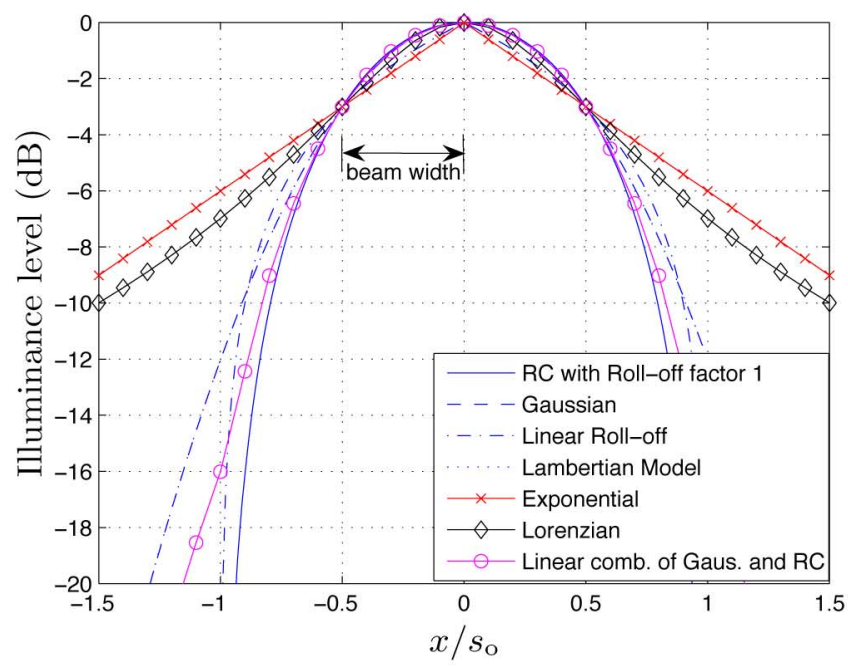

(a) Center fitting criterion.

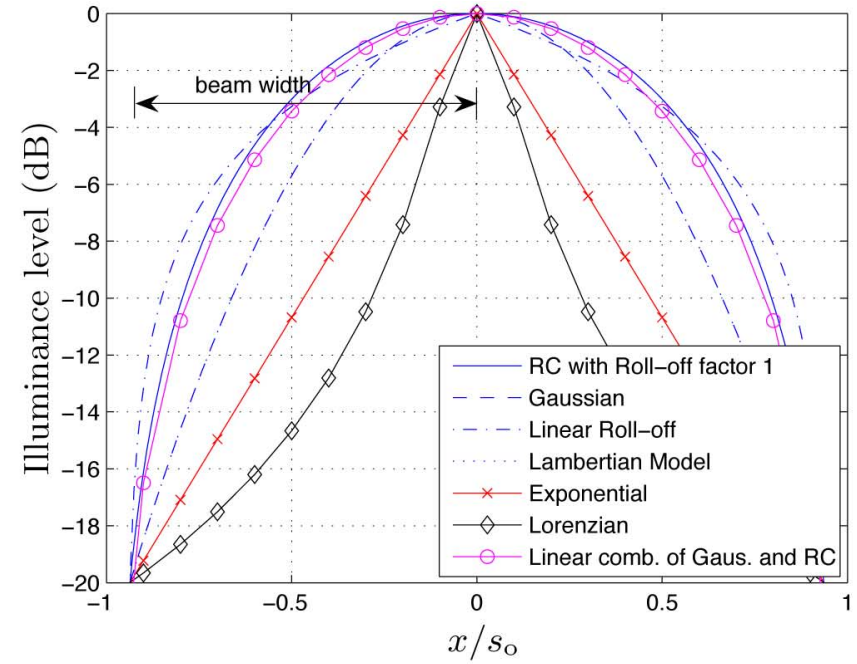

(b) Edge fitting criterion.

Fig. 6. Illustration of a few basic illumination patterns with the same beam width for two different criteria.

of the proposed basic patterns, we consider the tradeoff between rendering of uniform illumination patterns and the granularity in rendering of localized illumination patterns. For the performance of rendering uniform illumination patterns, we focus on the range of distances which are larger than a target distance $h_{0}$. To make a fair comparison in terms of rendering of localized illumination patterns, we consider all the basic illumination patterns with the same beam width. We provide two criteria for the definition of beam width. In the first criterion, the beam width is defined as the distance between the LED location and the position along the LED grid where $f_{b}$ is reduced to half of its maximum value. Therefore, this criterion focuses on the central part of a beam and is named center fitting criterion. The other criterion focuses on the perceivable edge size of $f_{b}$, and the beam width is defined as the distance between the LED location and the position along the LED grid where $f_{b}$ is reduced by $20 \mathrm{~dB}$ (see Section IV-B). Hence this criterion is named edge fitting criterion. These two criteria represent two distinct cases of beam width definition. Results for other criteria can be obtained similarly.

As discussed in Section $\mathrm{V}-\mathrm{C}$, we only need to consider the case with roll-off factor $\beta=1$ in the family of raised-cosine (RC) functions. We also consider a few other functions. The one-dimensional illustrations of these functions are shown in Fig. 6(a) and (b), for the center fitting criterion and edge fitting criterion, respectively. The $x$-coordinate is normalized by $s_{O}$. This normalization is taken because the level of uniformity achieved is mostly dependent on how much overlap exists between the light beams of neighboring LEDs. Hence a meaningful definition of beam width should be in terms of $s_{O}$. From Fig. 6, there is no visible difference between the Gaussian pattern and the Lambertian pattern for the case when $h=2 \mathrm{~m}$, and $s_{o}=0.1 \mathrm{~m}$, which represents a normal indoor situation. Therefore, we will only consider the Gaussian pattern in the rest of the paper. The reason why this difference is dependent on $h$ and $s_{O}$ is because, as will be shown in [11], the difference depends quite strongly on the Lambertian mode number, which in turn depends on the beam width at $h$. The beam width is, as discussed above, normalized in terms of $s_{O}$. The curve corresponding to the weighted combination of Gaussian (Gaus) and raised cosine (RC) functions is explained later in Section V-E.

The RMSE in rendering uniform illumination by these basic patterns is depicted and compared in Fig. 7(a) and (b), for the center fitting criterion and the edge fitting criterion, respectively.

It can be seen that the RMSE for the raised-cosine and linear roll-off functions fluctuates when $h / h_{o}$ increases above one, although perfect uniformity can be achieved at $h_{o}$. In contrast, the RMSE for the exponential, Gaussian and Lorenzian functions keeps decreasing while $h / h_{o}$ increases above one, although perfect uniformity can not be achieved. Therefore we propose to use the worst case RMSE in the range of $h / h_{o} \geq 1$ in order to make a fair comparison of all the functions. The worst case RMSE corresponds to the most observable error. From Fig. 7, two interesting basic patterns are the Gaussian pattern and the RC pattern. Both yield low RMSE, and appear to be similar in the spatial distribution. This motivates us to further explore the possibility of linearly combining these two and other patterns.

\section{E. Optimization of $f_{b}$ Through Linear Combination}

In Section V-D, we compared a few known functions as basic illumination patterns. To further optimize $f_{b}$, we propose to introduce more basic patterns through linear combination of some of the known functions, i.e.

$$
\hat{f}_{b}=\sum_{i} \alpha_{i} f_{b i}
$$

where $\left\{f_{b i}\right\}$ are the presented basic patterns, $\alpha_{i} \geq 0$ for each $i$ and $\sum_{i} \alpha_{i}=1$ such that the beam widths of different patterns are all identical. The linear combination of different basic patterns can yield a tradeoff between the advantages and disadvantages of different patterns. Moreover, as an example, the Fourier transform of the Gaussian function or exponential function is always positive while that of the raised cosine functions 


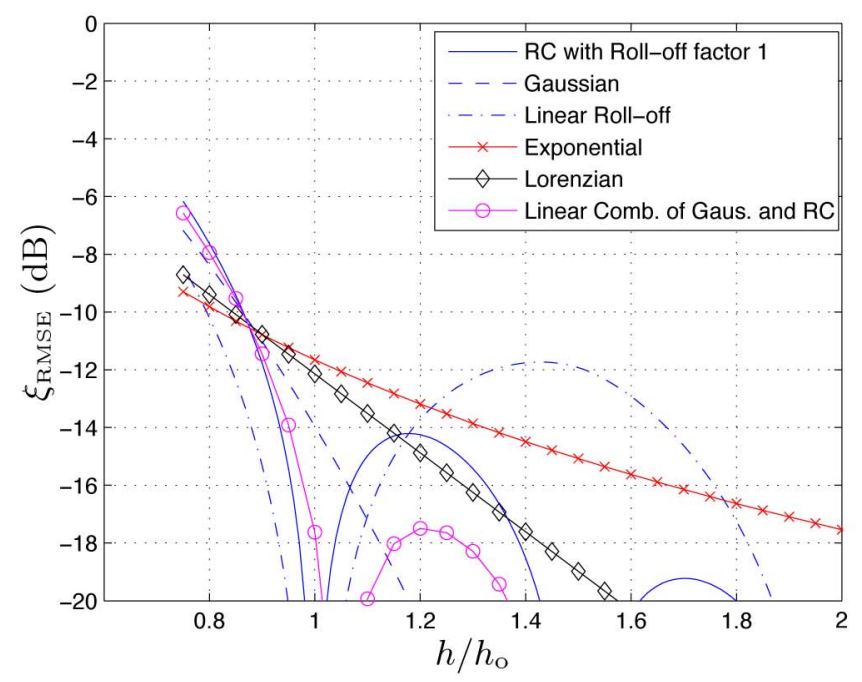

(a) Center fitting criterion.

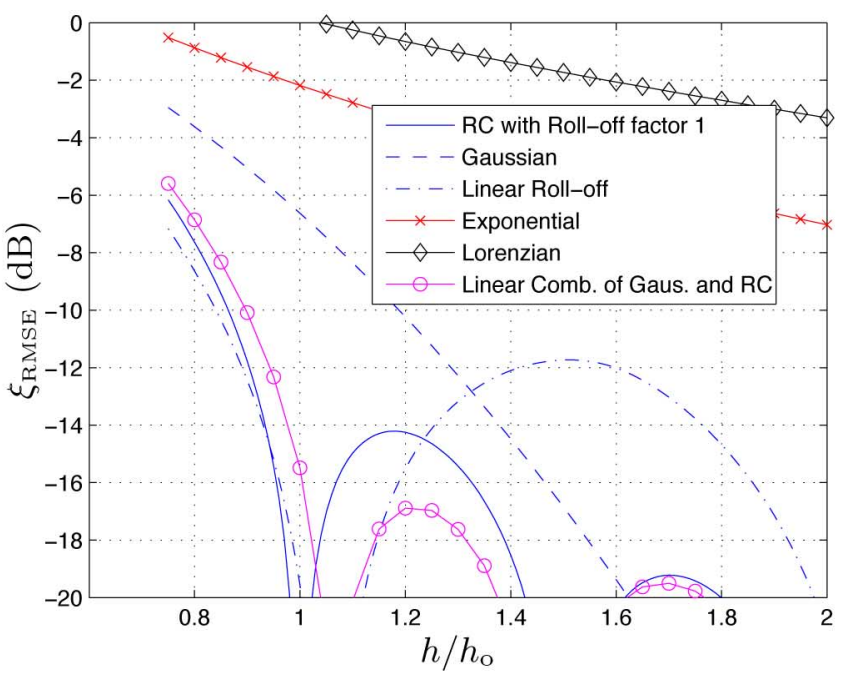

(b) Edge fitting criterion.

Fig. 7. RMSE in rendering uniform illumination by a regular LED array for different basic patterns with the same beam width based on two different criteria.

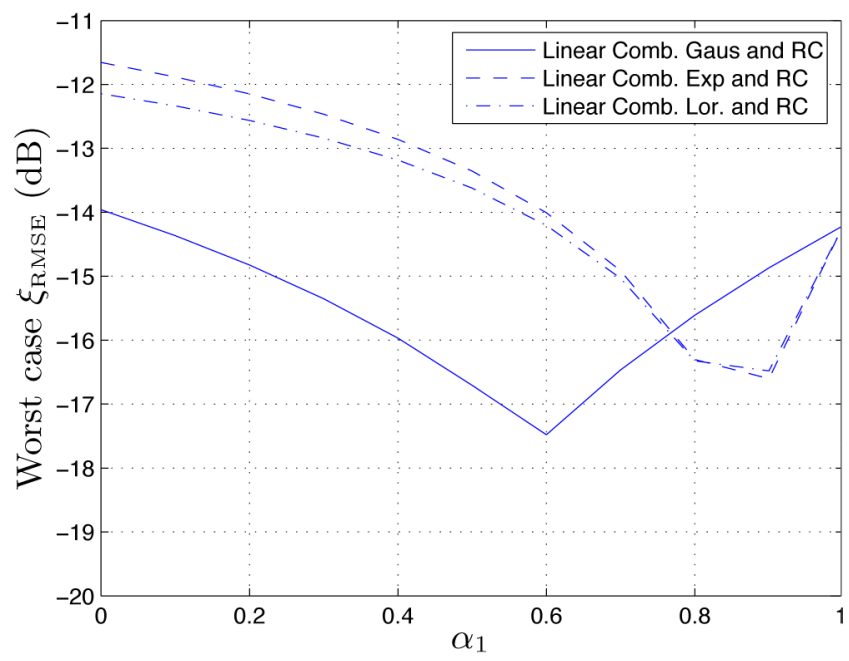

(a) Center fitting criterion.

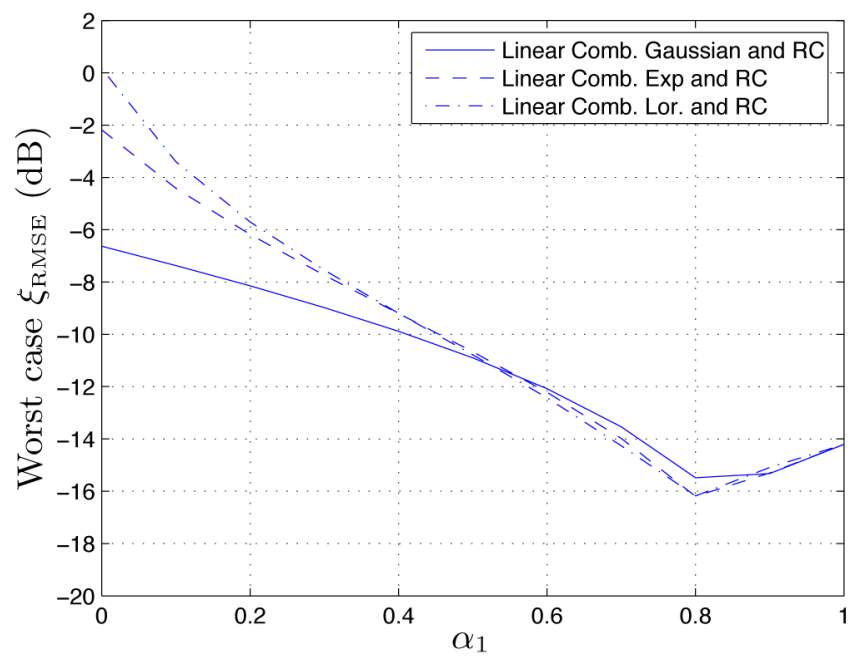

(b) Edge fitting criterion.

Fig. 8. Worst case RMSE in rendering uniform illumination by linear combinations of different basic patterns with identical beam widths.

may be positive or negative. Therefore, the linear combination of Gaussian and raised-cosine, or exponential and raised-cosine can perform even better due to possible cancelations.

The performances of different combinations, in terms of worse case RMSE for $h / h_{0} \geq 1$, are shown in Fig. 8(a) and (b), for the center fitting criterion and the edge fitting criterion, respectively. It shows that the performance of a linear combination of the raised-cosine and some other functions, such as the Gaussian, exponential and Lorentzian functions, can potentially be better than either of the original ones. Furthermore, for the center fitting criterion, it can be seen from Fig. 8(a) that the linear combination of the raised-cosine and Gaussian functions when $\alpha_{1}=0.6$, where $\alpha_{1}$ is the combination coefficient corresponding to the raised-cosine function, gives the best performance, which is more than $3 \mathrm{~dB}$ better than that given by either the Gaussian or raised-cosine pattern. The beam shape of this linear combination is also depicted in Fig. 6(a). We can observe that this function is very close to the raised-cosine function, except that its tails are heavier due to the Gaussian component. Similarly, for edge fitting criterion, Fig. 8(b) shows that the linear combination of raised-cosine and three other functions considered provide similar performances, and all perform best when $\alpha_{1}=0.8$. The best performance achieved is roughly 10 and $2 \mathrm{~dB}$ better than that given by the Gaussian and raised-cosine basic pattern, respectively.

It is illustrated in Fig. 6 that the Gaussian pattern is a very good two-dimensional approximation of the generalized Lambertian pattern of LEDs. In this section, we showed that the significantly better performance of uniform illumination rendering than that by the Gaussian basic pattern can be achieved by the proposed optimization approach. Moreover, the lowest RMSE obtained through this optimization is hardly perceivable, referring to Section IV-B. 


\section{F. Additional Discussions on Beam Widths and Beam Shapes}

In the above discussions, we compared a few basic patterns with identical beam widths. In particular, we chose the beam width to be $0.5 s_{o}$ for the center fitting criterion and $0.95 s_{o}$ for the edge fitting criterion, because these two beam widths correspond to the same raised-cosine pattern for which perfect uniformity is achieved at $h_{o}$ with minimum illumination granularity. For different scenarios, other values for beam widths can be chosen in order to compare different patterns. It turns out that there is a tradeoff between uniform illumination rendering and illumination granularity.

For the Gaussian basic pattern, increasing beam width, which is equivalent to increasing $h / h_{o}$ due to Property 1 , in Fig. 7(a), results in lower RMSE at the cost of larger illumination granularity. When the beam width for the center fitting criterion is increased to $0.6 s_{o}$, the RMSE is no longer perceivable. For the raised-cosine pattern and the weighted combination of the Gaussian and raised-cosine pattern, on the other hand, increasing the beam width from $s_{O}$ to $0.6 s_{O}$ does not result in any change in the worst case RMSE, while the rendered illumination granularity is increased. Similar observations for other basic patterns and for the edge fitting criterion can also be drawn from Fig. 7.

In this section, we discussed the performance of different basic patterns to provide guidelines for the design of LED illumination modules. In practice, these patterns can be implemented or approximated through collimating optics [16], [17]. For instance, due to the rapid development of solid state lighting technologies, there appears great variety of basic illumination patterns [7], [8]. Therefore, it would be easy to implement the basic illumination patterns proposed in this paper, however discussions on the details of such optics are beyond the scope of this paper. Furthermore, the collimating optics are located near the LEDs, and at the far field, the combined effect from the LEDs and the optics can be still treated as that from a point source. Therefore, all the results presented above, e.g., the scaling property, are still valid in the presence of such collimating optics.

Further, we only consider the lighting system with a larger number of LEDs in this paper. When the number of LEDs is small, especially when the edge effects play a significant role, we might have certain different observations, e.g., the monotonic behavior for the uniformity achieved through the Gaussian basic pattern may not hold, [5]. Nevertheless, as
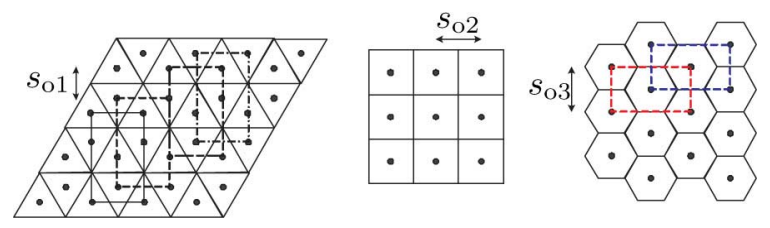

Fig. 9. Triangular, square, and hexagonal grid shapes.

shown in Section I, for the application scenario considered in this paper, the edge effects are negligible, hence we will not provide further discussions on the edge effects in this paper.

Moreover, given the regular LED array considered for the convenience of mass production, we do not consider significant differences among the LEDs in terms of peak intensity, color and angular distribution, etc., which can be solved by binning operations [18] during production, in a future lighting system.

Finally, we focus on an analytical approach, instead of another widely used approach of Monte Carlo simulation, when evaluating the performance of illumination rendering in this paper. This is because we consider uniform illumination rendering by an infinite LED array to neglect the edge effects. In this case, the Monte Carlo method is not applicable. Further, the analytical approach allows an efficient evaluation of the performances achieved at different distances (see (15)), which can not be as efficiently accomplished by the Monte Carlo method.

\section{COMPARISON OF TRIANGUlar, SQUARE AND HEXAGONAL GRID SHAPES}

In previous sections, we presented a framework for evaluating the performance of illumination rendering by a square (rectangular) array of LEDs. In this section, we extend the framework to other regular LED grid shapes and compare their results with that of the rectangular grid shape. For uniform illumination rendering, it is desirable to have a regular LED grid shape with rotational symmetry. It is well known that we only need to consider three such basic grid shapes. We show this result in Lemma 3 and a simple proof is presented in Appendix II.

Lemma 3: For a two-dimensional regular grid which covers the entire space, if it is symmetric under rotations over an angle $\psi$, where $0<\psi<\pi$, then the regular grid must be either triangular, square or hexagonal. ${ }^{3}$

${ }^{3}$ The grid shapes, as illustrated in Fig. 9, are named according to the reciprocal shapes of the LED lattice.

$$
\begin{aligned}
\xi_{\text {RMSE-TRI }} & =\frac{1}{P_{b}}\left[\sum_{\left\{l, n \mid l^{2}+n^{2} \neq 0\right\}}\left|F_{b}\left(l \frac{r_{h}}{3 s_{o 1}}, n \frac{r_{h}}{\sqrt{3} s_{o 1}}\right)\left(1+(-1)^{l+n}\right) \sqrt{2+2 \cos \left(\left(l+\frac{n}{3}\right) \pi\right)}\right|^{2}\right]^{(1 / 2)} \\
\xi_{\text {RMSE-HEX }} & =\frac{1}{P_{b}} \sqrt{\sum_{\left\{l, n \mid l^{2}+n^{2} \neq 0\right\}}\left|F_{b}\left(l \frac{r_{h}}{s_{o 3}}, n \frac{r_{h}}{\sqrt{3} s_{o 3}}\right)\left(1+(-1)^{l+n}\right)\right|^{2}}
\end{aligned}
$$




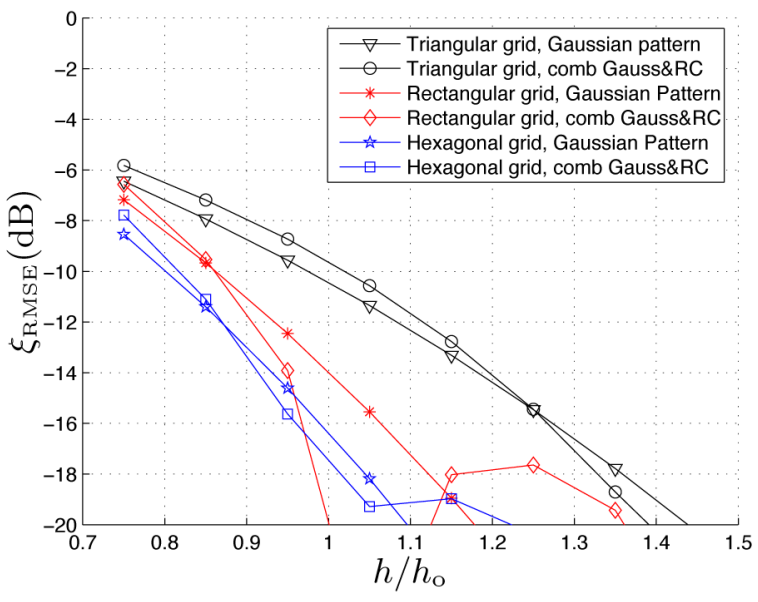

(a) Center fitting criterion.

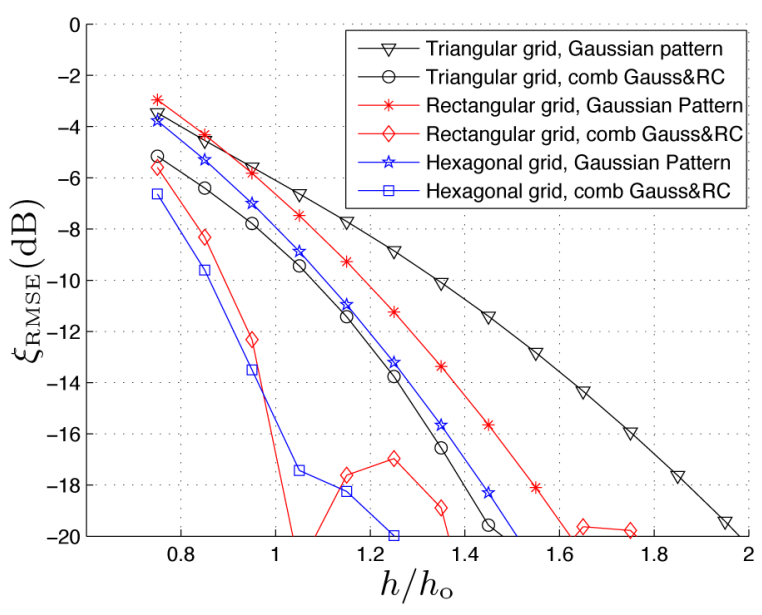

(b) Edge fitting criterion.

Fig. 10. Comparison RMSE versus $h / h_{o}$ for the triangular, square, and hexagonal grid shapes.

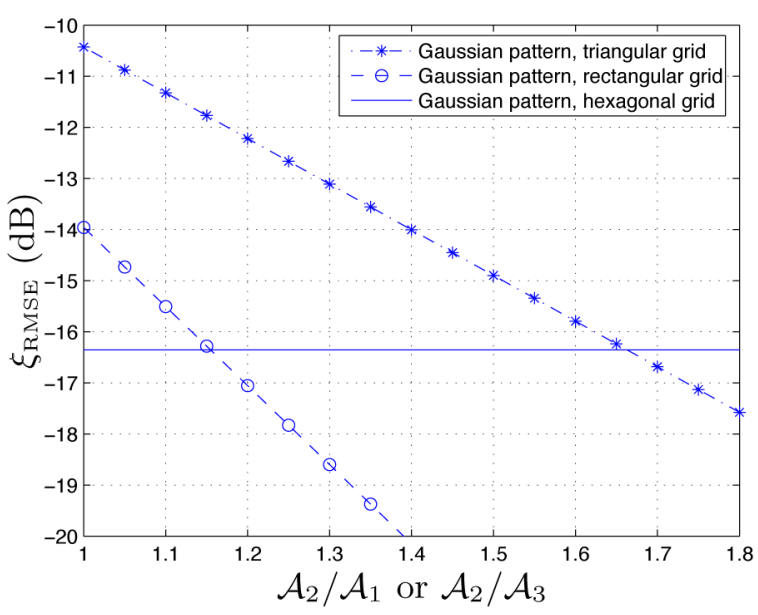

(a) Center fitting criterion.

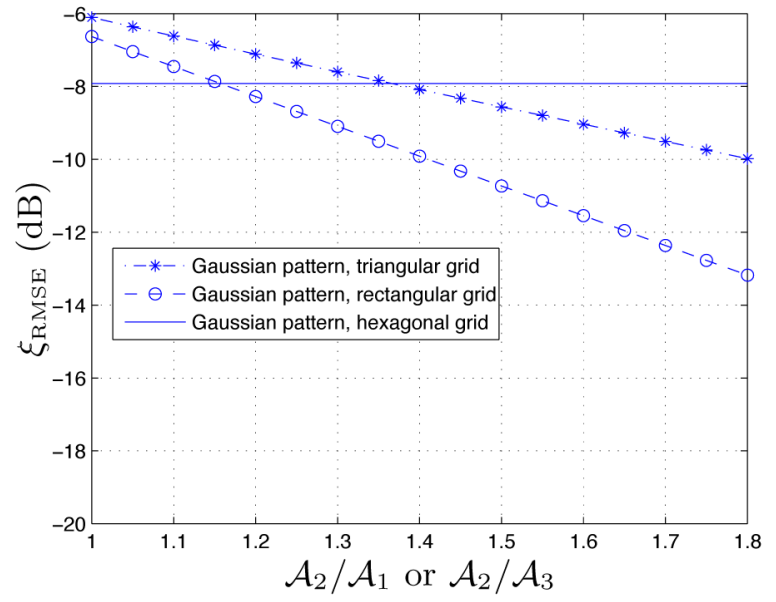

(b) Edge fitting criterion.

Fig. 11. RMSE versus relative densities of triangular and square LED arrays with respect to that of hexagonal array.

Fig. 9 depicts the three grid shapes, where the minimum distances between two LEDs are denoted as $s_{o 1}, s_{o 2}$, and $s_{o 3}$, respectively. In this section, the performances of the three grid shapes are discussed and compared. To make a fair comparison, we assume the LED densities and the basic illumination patterns are the same for the three grid shapes. The area of each basic component grid can be obtained as $\left\|\mathcal{A}_{1}\right\|=(3 \sqrt{3} / 4) s_{o 1}^{2}$, $\left\|\mathcal{A}_{2}\right\|=s_{o 2}^{2}$, and $\left\|\mathcal{A}_{3}\right\|=(\sqrt{3} / 2) s_{o 3}^{2}$, respectively, and hence $s_{o 1}^{2}=(4 /(3 \sqrt{3})) s_{o 2}^{2}=(2 / 3) s_{o 3}^{2}$.

From Fig. 9, the LED array with triangular and hexagonal grids can be seen as consisting of four and two LED arrays with rectangular grids, respectively. Hence, the RMSEs for these two grids can be obtained similarly as was done in the previous sections. More specifically, we compute the RMSE in the unit region of any of the component rectangular grid for the sake of convenience and the results are equivalent, because the unit rectangular region consists of multiple unit hexagonal or triangular grids. Eventually, we get that the RMSE for the triangular and hexagonal grid are shown in (26)-(27) at the bottom of the previous page. We only consider two basic illumination patterns, i.e., the Gaussian pattern and the linear combination of the Gaussian and raised-cosine pattern (see Section V-E), in order to compare the RMSE for the three grid shapes. These two basic patterns correspond to the spontaneous LED illumination pattern and the best basic pattern we have obtained for the square grid respectively. The RMSE for different grid shapes is depicted in Fig. 10(a) and (b) for the center and edge fitting criterion, respectively. In Fig. 10(a), when the basic illumination pattern is Gaussian, the RMSE for the hexagonal grid is roughly $2 \mathrm{~dB}$ lower than that for the square grid, which in turn is roughly $3 \mathrm{~dB}$ lower than that for the triangular grid. The linear combination of the Gaussian and raised-cosine is applied with $\alpha_{1}=0.6$ and $\alpha_{1}=0.8$ for the center fitting criterion and edge fitting criterion, respectively. For this basic illumination pattern, the same order of performance, in terms of worst case RMSE, still holds. This order of performances is reasonable in the sense that the number of neighboring LEDs with the smallest distance varies from three to four and five, when the grid varies from triangular into square and hexagonal. Similar observations can also be made from Fig. 10(b). 
This means that a more uniform illumination effect can be achieved by arranging the same number of LEDs in the hexagonal rather than in the square or the triangular grid. In other words, if we only need to obtain a desired level of uniformity, we can decrease the number of LEDs to reduce the cost, or reduce the beam width of the LEDs to provide better granularity in rendering other patterns.

To further quantify the advantage of the hexagonal grid, we can also compare the LED densities at the same RMSE performance. The performance of uniform pattern rendered by a square and a triangular LED array as the LED density increases, is shown in Fig. 11(a), where the beam width of the Gaussian pattern is equal to the raised-cosine pattern in terms of the center fitting criterion. As a reference, the RMSE rendered by a hexagonal LED array at height $h_{o}$ is also shown in Fig. 11(a). It is shown that $(1.15-1) / 1.15=13 \%$ and $(1.65-1) / 1.65=39 \%$ LEDs can be saved if we use a hexagonal grid instead of a square and triangular grid to achieve the same performance in terms of RMSE. For the Gaussian pattern whose beam width is equal to the raised-cosine pattern in terms of the edge fitting criterion, similar conclusions can be drawn from Fig. 11(b), except that $13 \%$ and $27 \%$ LEDs can be saved in this case, if the hexagonal grid is used instead of the square and triangular grid, respectively. Note that similar insights can also be obtained for finite number of LEDs in [5], and for the sampling and reconstruction of 2-D signals in [19], [20]. This fact illustrates that the results and insights obtained in this paper can be also widely applied to other different practical scenarios.

\section{CONCLUSION}

In this paper, a mathematical framework is presented to investigate illumination rendering by an array of LEDs, and guidelines are provided to design an LED illumination system. We focus on uniform illumination rendering by LEDs with narrow beams and analyze performance in terms of the proposed cost function, the RMSE. We proved that maximum uniformity in illumination rendering can be achieved by setting the intensity levels of all the LEDs to be identical. We also presented the scaling rule of the basic illumination pattern with respect to the distance from the ceiling and thus proposed a convenient approach to evaluate the performance of uniform illumination rendering across a range of distances. This scaling property is also potentially useful for practical engineers when designing other nonuniform patterns. Through the design of the basic illumination pattern of a single LED, we conclude that significantly better performance can be achieved, using the proposed weighted combination of Gaussian and the raised-cosine function instead of the conventional Lambertian function. The residual rendering error achieved due to this proposed basic pattern is hardly perceivable. Note that the patterns discussed and proposed in this paper can be implemented or approximated through collimating optics. Moreover, through comparing three different LED grid shapes, namely the triangular, square and hexagonal grids, we not only confirm the intuition that hexagonal grid shape is better, but also conclude numerically that better uniform illumination pattern, 2 and $5 \mathrm{~dB}$ in terms of RMSE, can be achieved by using hexagonal, instead of square or triangular grid. Alternatively, $13 \%$ and 39\% LEDs can be saved if hexagonal grid is used instead of square and triangular, respectively, while achieving identical granularity in localized illumination rendering and identical performance in uniform illumination rendering. A potential direction for further study would be to investigate localized illumination rendering through an array of LEDs.

\section{APPENDIX I \\ DEDUCTION OF (12)}

In this appendix, we give a detailed deduction for (12). See (28)-(31) at the bottom of the page, where (30) and (31) follow from the first and last equation of (10), respectively.

Now, consider the fact that the sequence $\left\{F_{b}\left(l\left(1 / s_{o}\right), n\left(1 / s_{o}\right) ; h\right)\right\}$, where $l$ and $n$ are integers,

$$
\begin{aligned}
\xi_{\mathrm{RMSE}}(h)= & {\left[\frac{1}{f_{t}^{2}} \frac{1}{s_{o}^{2}} \int_{-s_{o} / 2}^{s_{o} / 2} \int_{-s_{o} / 2}^{s_{o} / 2}\left(\sum_{l=-\infty}^{\infty} \sum_{n=-\infty}^{\infty} v_{c} f_{b}\left(x-l s_{o}, y-n s_{o} ; h\right)-f_{t}\right)^{2} \mathrm{~d} x \mathrm{~d} y\right]^{1 / 2} } \\
= & {\left[\frac{1}{f_{t}^{2}} \frac{1}{s_{o}^{2}} v_{c}^{2} \int_{-s_{o} / 2}^{s_{o} / 2} \int_{-s_{o} / 2}^{s_{o} / 2}\left(\sum_{l=-\infty}^{\infty} \sum_{n=-\infty}^{\infty} f_{b}\left(x-l s_{o}, y-n s_{o} ; h\right)\right)^{2} \mathrm{~d} x \mathrm{~d} y\right.} \\
& \left.-2 \frac{1}{f_{t}^{2}} \frac{1}{s_{o}^{2}} v_{c} f_{t} \int_{-s_{o} / 2}^{s_{o} / 2} \int_{-s_{o} / 2}^{s_{o} / 2} \sum_{l=-\infty}^{\infty} \sum_{n=-\infty}^{\infty} f_{b}\left(x-l s_{o}, y-n s_{o} ; h\right) \mathrm{d} x \mathrm{~d} y+1\right]^{1 / 2} \\
= & {\left[\frac{1}{f_{t}^{2}} \frac{1}{s_{o}^{2}} v_{c}^{2} \int_{-s_{o} / 2}^{s_{o} / 2} \int_{-s_{o} / 2}^{s_{o} / 2}\left(\sum_{l=-\infty}^{\infty} \sum_{n=-\infty}^{\infty} f_{b}\left(x-l s_{o}, y-n s_{o} ; h\right)\right)^{2} \mathrm{~d} x \mathrm{~d} y-1\right]^{1 / 2} } \\
= & {\left[\frac{s_{o}^{2}}{P_{b}^{2}} \int_{-s_{o} / 2}^{s_{o} / 2} \int_{-s_{o} / 2}^{s_{o} / 2}\left(\sum_{l=-\infty}^{\infty} \sum_{n=-\infty}^{\infty} f_{b}\left(x-l s_{o}, y-n s_{o} ; h\right)\right)^{2} \mathrm{~d} x \mathrm{~d} y-1\right]^{1 / 2} }
\end{aligned}
$$




$$
\begin{aligned}
\sum_{l, n}\left|F_{b}\left(l \frac{1}{s_{o}}, n \frac{1}{s_{o}} ; h\right)\right|^{2} & =\frac{1}{s_{o}^{2}} \int_{-s_{o} / 2}^{s_{o} / 2} \int_{-s_{o} / 2}^{s_{o} / 2}\left(\sum_{l=-\infty}^{\infty} \sum_{n=-\infty}^{\infty} s_{o}^{2} f_{b}\left(x-l s_{o}, y-n s_{o} ; h\right)\right)^{2} \mathrm{~d} x \mathrm{~d} y \\
& =s_{o}^{2} \int_{-s_{o} / 2}^{s_{o} / 2} \int_{-s_{o} / 2}^{s_{o} / 2}\left(\sum_{l=-\infty}^{\infty} \sum_{n=-\infty}^{\infty} f_{b}\left(x-l s_{o}, y-n s_{o} ; h\right)\right)^{2} \mathrm{~d} x \mathrm{~d} y
\end{aligned}
$$

and $\sum_{l=-\infty}^{\infty} \sum_{n=-\infty}^{\infty} s_{o}^{2} f_{b}\left(x-l s_{o}, y-n s_{o} ; h\right)$ where $-s_{o} / 2 \leq x, y \leq s_{o} / 2$ consist of a two-dimensional Fourier pair in terms of discrete-time Fourier transform, we have [see (32) at the top of the page] from the Parseval's theorem. The two-dimensional form of the Parseval's theorem is a simple extension of the one-dimensional form [21].

Substituting (32) into (30), we get

$$
\begin{aligned}
\xi_{\mathrm{RMSE}}(h) & =\left[\frac{1}{P_{b}^{2}} \sum_{l, n}\left|F_{b}\left(l \frac{1}{s_{o}}, n \frac{1}{s_{o}} ; h\right)\right|^{2}-1\right]^{1 / 2} \\
& =\frac{1}{P_{b}} \sqrt{\sum_{\left\{l, n \mid l^{2}+n^{2} \neq 0\right\}}\left|F_{b}\left(l \frac{1}{s_{o}}, n \frac{1}{s_{o}} ; h\right)\right|^{2}}
\end{aligned}
$$

where the last equation follows from the fact that $F_{b}(0,0 ; h)=$ $P_{b}$ that can be derived from (13).

\section{APPENDIX II \\ PROOF OF LEMMA 3}

Proof: Set a point on a grid as the origin with the coordinates $(0,0)$. We can take a vector, connecting this point to one of its neighboring points on the grid. Let this vector be represented as $[1,0]^{T}$, where $(\cdot)^{T}$ denotes the transpose operation.

Due to the property of rotational symmetry, there is another vector $[\cos \psi, \sin \psi]^{T}$, connecting this point to another neighboring point on the grid. These two vectors can be taken as a set of bases for the two dimensional grid, any other point on the grid can be written as integer combination of these two vectors.

Also due to the property of rotational symmetry, there must be another vector $[\cos 2 \psi, \sin 2 \psi]^{T}$, where $0<\psi<\pi$, connecting the origin to the third neighboring point on the grid. Hence, we know that

$$
[\cos 2 \psi, \sin 2 \psi]^{T}=x_{1}[1,0]^{T}+x_{2}[\cos \psi, \sin \psi]^{T}
$$

where $x_{1}$ and $x_{2}$ are both integers. Hence, we get $\sin 2 \psi=$ $x_{2} \sin \psi$, which can be simplified into $\cos \psi=x_{2} / 2$. Since $-1<\cos \psi<1, x_{2}$ can be only $-1,0$, or 1 , and $\psi$ can be, thus, only be $2 \pi / 3, \pi / 2$, or $\pi / 3$, which in turn results into triangular, square, or hexagonal grid, respectively.

\section{ACKNOWLEDGMENT}

The authors would like to acknowledge valuable discussions with C. K. Ho and A. Pandharipande. The authors would also like to thank the associate editor Prof. B. L. Evans and anonymous reviewers for their valuable comments and suggestions that greatly helped to improve the manuscript.

\section{REFERENCES}

[1] S. Muthu, F. J. P. Schuurmans, and M. D. Pashley, "Red, green, and blue LEDs for white light illumination," IEEE J. Sel. Topics Quantum Electron., vol. 8, pp. 333-338, Mar./Apr. 2002.

[2] G. Pang, T. Kwan, H. Liu, and C.-H. Chan, "LED wireless," IEEE Ind. Appl. Mag., vol. 8, no. 1, pp. 21-28, Jan./Feb. 2002.

[3] LUXEON LEDs:: High Power LEDs LUMILEDS [Online]. Available: http://www.lumileds.com/products/luxeon/

[4] I. Moreno, J. Muñoz, and R. Ivanov, "Uniform illumination of distant targets using a spherical light-emitting diode array," Opt. Eng., vol. 46, Mar. 2007.

[5] I. Moreno, M. Avendaño-Alejo, and R. I. Tzonchev, "Designing lightemitting diode arrays for uniform near-field irradiance," Appl. Opt., vol. 45, pp. 2265-2272, Apr. 2006.

[6] I. Moreno and R. I. Tzonchev, "Effects on illumination uniformity due to dilution on arrays of LEDs," in Proc. SPIE, 2004, vol. 5529, pp. 268-275.

[7] I. Moreno and U. Contreras, "Color distribution from multicolor LED arrays," Opt. Express, vol. 15, pp. 3607-3618, Mar. 2007.

[8] I. Moreno, C.-Y. Tsai, D. Bermũdez, and C.-C. Sun, "Simple function for intensity distribution from LEDs," in Proc. SPIE, 2007, vol. 6670.

[9] L. Svilainis and V. Dumbrava, "LED far field pattern approximation performance study," in Proc. Int. Conf. on Inf. Technol. Interfaces, 2007, pp. 645-649.

[10] D. Vincenzi and G. Oliva, "Tailoring freeform lenses for barcode lighting," in Proc. IEEE Workshop on Autom. Identif. Adv. Technol., 2007, pp. 134-139.

[11] H. Yang, J. W. M. Bergmans, T. C. W. Schenk, J.-P. M. G. Linnartz, and R. Rietman, "An analytic model for the illuminance distribution of a power LED," Opt. Express, vol. 16, no. 26, pp. 21641-21646, Dec. 2008.

[12] J. R. Barry, Wireless Infrared Communications. Boston, MA: Kluwer Academic, 1994

[13] P. R. Boyce, Human Factors in Lighting, 2nd ed. New York: Taylor and Francis, 2003.

[14] V. Jungnickel, V. Pohl, S. Nönnig, and C. V. Helmolt, "A physical model of the wireless infrared communication channel," IEEE J. Sel Areas Commun., vol. 20, pp. 631-640, Apr. 2002.

[15] J. G. Proakis, Digital Communications. New York: McGraw-Hill, 2000, ch. 9.

[16] R. Winston, J. C. Minano, and P. G. Benitez, Nonimaging Optics. New York: Academic, 2004.

[17] The Optical Plastics Specialists Polymer Optics Ltd. [Online]. Available: http://www.polymer-optics.co.uk/

[18] Binning and Labeling LUMILEDS [Online]. Available: http://www. lumileds.com/pdfs/AB21.PDF

[19] D. E. Dudgeon and R. M. Mersereau, Multidimensional Digital Signal Processing. Englewood Cliffs, NJ: Prentice-Hall, 1983, ch. 1.

[20] D. P. Petersen and D. Middleton, "Sampling and reconstruction of wave-number limited functions in N-dimensional Euclidean spaces," Inf. Control, vol. 5, pp. 279-323, 1962.

[21] J. R. Barry, E. A. Lee, and D. G. Messerschmitt, Digital Communication, 3rd ed. Norwell, MA: Kluwer, 2003, ch. 2. 


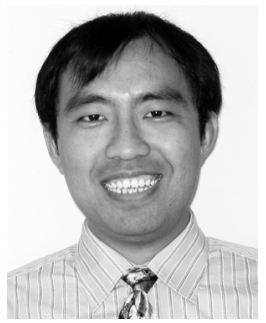

Honging Yang (S'06) received the B.S. and M.S. degrees from the Department of Electronic Engineering, Tsinghua University, Beijing, China, in 2000 and 2003, respectively. He also received the M.E. degree from the Department of Electrical and Computer Engineering, National University of Singapore, Singapore, in 2005.

$\mathrm{He}$ is now working toward the Ph.D. degree with Eindhoven University of Technology (TU/e), Eindhoven, The Netherlands, while conducting joint research with Philips Research Laboratories, Eindhoven. His research interest lies in signal processing for illumination systems, digital communications, and recording systems.

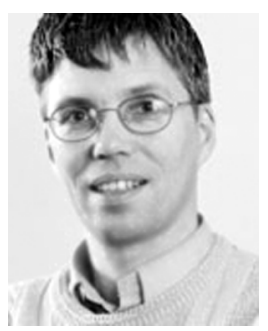

Jan W. M. Bergmans (SM'91) received the degree of Elektrotechnisch Ingenieur (cum laude) in 1982, and the Ph.D. degree in 1987, both from Eindhoven University of Technology, Eindhoven, The Netherlands.

From 1982 to 1999, he was with Philips Research Laboratories, Eindhoven, working on signal-processing techniques and IC-architectures for digital transmission and recording systems. In 1988 and 1989, he was an exchange researcher with Hitachi Central Research Labs, Tokyo, Japan. Since 1999, he has been Professor and Chairman of the Signal Processing Systems Group, Eindhoven University of Technology. Since 1998, he has been an advisor to the Data Storage Institute, Singapore, and since 2000, to Philips Research Laboratories, Eindhoven. He has published extensively in refereed journals, has authored a book, Digital Baseband Transmisison and Recording (Boston, MA: Kluwer Academic, 1996), and holds approximately 40 U.S. patents.

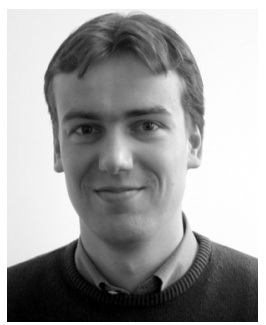

Tim C. W. Schenk (S'01-M'07) received the M.Sc. and Ph.D. degrees in electrical engineering from Eindhoven University of Technology (TU/e), Eindhoven, The Netherlands, in 2002 and 2006, respectively.

From 2002 to 2004, he was with the Wireless Systems Research Group, Agere Systems, Nieuwegein, The Netherlands. From 2004 to 2006, he was a Research Assistant with the Radiocommunications Group, TU/e. Since October 2006, he has with Philips Research Laboratories, Eindhoven, as a Senior Scientist and Cluster Leader in the Distributed Sensor Systems Department. His current research interests include applied signal processing, lighting controls, and wireless optical communications. He authored the book RF Imperfections in High-Rate Wireless Systems: Impact and Digital Compensation (The Netherlands: Springer, 2008).

Dr. Schenk was awarded the 2006 Veder Award from the Dutch Scientific Radio Fund Veder for his contributions in the field of system optimization for multiple-antenna systems.

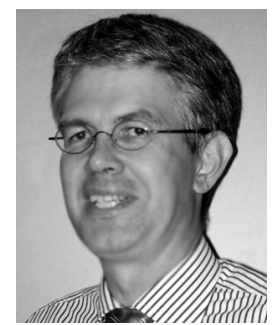

Jean-Paul M. G. Linnartz (S'85-M'87-SM'99) received the M.Sc. degree (cum laude) from Eindhoven University of Technology (TU/e), Eindhoven, The Netherlands, in 1986, and the Ph.D. degree (cum laude) from Delft University of Technology (T.U. Delft), The Netherlands, in December 1991.

$\mathrm{He}$ is a Senior Technology Director with Philips Research, Eindhoven, where he is responsible for research in the domain of sensor networks and systems. Previously, he headed security and connectivity research groups. He is also a part-time professor with TU/e. He joined Philips in 1995, initially to set up a research activity on digital rights management for multimedia content and security systems. He applied signal detection principles in the field of electronic watermarking, and invented various attacks and security measures. He introduced a privacy-preserving method of biometric identification that prevents misuse of templates from data bases. He proposed algorithms to mitigate Doppler intercarrier interference in OFDM reception for NXP chip sets for mobile DVB television reception. He introduced coded light, a modulation scheme that allows the embedding of identifiers in light sources, to facilitate convenient control of illumination. He holds 25 patents. During 1992-1995, he was an Assistant Professor with the University of California at Berkeley. In 1993, he proposed and analyzed multicarrier CDMA. From 1988 to 1991 and in 1994, he was Assistant and Associate Professor with T.U. Delft, respectively.

Ronald Rietman received the Ph.D. degree in theoretical physics from the University of Amsterdam, The Netherlands, in 1993.

He was a Postdoctoral Fellow with the Theoretical Physics Group, Norwegian Institute of Technology, Trondheim, Norway, from 1993 to 1995. Since 1995, he has been with Philips Research Laboratories, Eindhoven, The Netherlands, where he has worked on a variety of topics such as electromagnetic compatibility, performance analysis of medium access control protocols, and PHY and MAC layer for $60-\mathrm{GHz}$ wireless communication. 